\title{
Lidil
}

Revue de linguistique et de didactique des langues

45 | 2012

Pratiques de formation à la lecture-écriture des adultes en parcours d'insertion

\section{Benjamin Joinau et Frédérique Pénilla (dir.), Universalisme et Multiculturalisme}

éd. Mukga, Séoul, Croisement, $n^{\circ}$ 1, 2011

\section{Soulisack Luanglad}

\section{OpenEdition \\ Journals}

Édition électronique

URL : http://journals.openedition.org/lidil/3227

DOI : 10.4000/lidil.3227

ISSN : 1960-6052

Éditeur

UGA Éditions/Université Grenoble Alpes

\section{Édition imprimée}

Date de publication : 15 mai 2012

Pagination : 182-184

ISBN : 978-2-84310-226-4

ISSN : $1146-6480$

Référence électronique

Soulisack Luanglad, «Benjamin Joinau et Frédérique Pénilla (dir.), Universalisme et Multiculturalisme », Lidil [En ligne], 45 | 2012, mis en ligne le 15 novembre 2013, consulté le 23 septembre 2020. URL http://journals.openedition.org/lidil/3227 ; DOI : https://doi.org/10.4000/lidil.3227 


\section{Notes de lecture}

Benjamin Joinau, Frédérique Pénilla, (dir.), Universalisme et

Multiculturalisme, éd. Mukga, Séoul, Croisement, n 1, 2011, 243 p.

Il s'agit de la revue Croisement $\mathrm{n}^{\circ} 1$, revue francophone de sciences humaines d'Asie de l'Est. Cette première livraison est constituée de 16 articles auxquels ont contribué des chercheurs coréens, chinois, japonais et occidentaux qui ont voulu, chacun dans sa spécialité, souligner et comparer les points spécifiques de leurs domaines, entre pays d'Asie de l'Est et France.

Dominic Thomas de l'université de Californie, Los Angeles, fait le point sur les littératures postcoloniales et la mondialisation. Dans «Littérature du monde en français », il s'interroge sur les différences et les similarités culturelles, économiques, politiques et sociales et leur influence sur le plan linguistique.

Gilles Dupuis de l'université de Montréal (Canada), s'intéresse aux œuvres de l'écrivaine canadienne d'origine chinoise, Ying Chen, et se demande si la littérature migrante est universelle.

Lianxing Gai, professeur de français à l'université de communication de Chine et Mingli Wang, professeur et doyen du Département de français du Beijing International Studies University (Chine), soulignent l'intérêt de la recherche en langue et culture, dans les discours publicitaires des produits et des noms des entreprises françaises en Chine. Pour eux la bonne traduction dans la publicité consiste à trouver des équivalences et correspondances d'un système à l'autre, en tenant compte de l'esprit du peuple local.

Lihua Zheng de l'université des Études étrangères du Guangdong (Chine), fait une comparaison entre la signature chinoise et la signature occidentale. Dans son article, il donne une explication sur le choix de la langue pour la signature chez les cadres chinois (certains choisissent de signer en chinois et d'autres avec l'alphabet latin), la constance $v s$ non-constance, et la lisibilité vs illisibilité de la signature : en effet, lorsqu'ils écrivent en caractères chinois, ceux-ci peuvent varier en style ou en régularité et présentent parfois des formes bien différentes. Pour lui les différences de signature entre la Chine et l'Occident sont le reflet de différences culturelles.

De son côté, Jinling Zhang de l'Institut d'études européennes, académie des sciences sociales de Chine, analyse historiquement les images dominantes de la Chine en France.

Yugang Liu, Rixuan Xu et Xiuru Niu soulignent l'intérêt de la théorie de l'Espace mental de G. Fauconnier, ils mènent une réflexion 
sur les propriétés cognitives et culturelles du transfert psychologique dans la communication.

Hui Cao de l'université de Jiao Tong de Shanghai (Chine), diagnostique les genèses culturelles et l'universalisme des Droits de l'Homme dans le cadre de la pensée occidentale et les compare avec celles de la Chine.

L'article de Kiyonobu Date, de l'université Tohoku Fukushi (Japon), présente une comparaison entre la laïcisation le développement des Droits de Homme au Japon.

Noriyuki Nishiyama de l'université de Kyoto (Japon), étudie la problématique du développement d'une politique coloniale d'assimilation homogène durant sa phase d'expansion territoriale, afin d'assimiler les indigènes à l'image de la France, dans le cadre du mouvement colonial français de la Troisième République, à partir des positions de deux idéologues : Gustave Le Bon et Léopold de Saussure.

Kenji Kanno, de l'université des Sciences de Tokyo (Japon), fait des remarques sur l'œuvre romanesque de Kikou Yamata, dans les relations culturelles japono-françaises.

Aya Ito de l'université Waseda, Tokyo (Japon), analyse les usages de l'iki (expression d'une permanente dualité, dont l'Orient et Occident sont deux pôles incommensurables) dans le monde multiculturel de Shûzô Kuki.

L'article de Yun Ch'Oe de l'université Sogang, Séoul (Corée), pose la question de l'universalité et du spécifique dans le domaine de la littérature. Il propose un nouvel angle pour penser les littératures spécifiques, minoritaires, telles que les littératures francophones.

Antoine Coppola, de l'université Sungkyunkwan, Séoul (Corée), note l'influence du roman L'Étranger d'A. Camus dans la littérature et le cinéma mondial, en particulier dans deux films coréens dans lesquels les thèmes et archétypes camusiens sont transposés dans l'espace et dans le temps.

Yves Millet, de l'université Hankuk, Séoul (Corée), fournit des outils d'analyse de la performance artistique; il a choisi de reconsidérer sous l'angle de l'hylémorphisme les réalisations de groupes japonais.

Gutai Hisashi Fujita, de l'université Kyushu-Sangyo (Japon), présente la «métaphore », « analogie » ou « image » que Bergson impose comme quelque chose d'indispensable à la compréhension de sa philosophie, dont la «voie», le «feu » et le « chemin », dans son dernier ouvrage intitulé Les deux sources de la morale et de la religion. 
Jean-François Graziani, de l'université de Kyoto (Japon), présente les points de vue de Dominique de Villepin, ancien Premier ministre français, de Keishi Saeki de l'université de Kyoto, de Nobutaka Miura de l'université Chuo de Tokyo, à l'occasion d'un discours au forum sur «La formation d'une vision du monde multipolaire et l'enseignement des langues étrangères », qui s'est tenu le 20 juin 2008 à l'université de Kyoto.

L'ouvrage dans son ensemble présente les résultats de travaux de recherche et les points de vue de spécialistes des divers domaines. Sa volonté de faire se confronter et se rencontrer, au-delà des discours, des cultures différentes, aboutit à une vraie réussite, qui questionne toutefois la soidisant unité d'une vision de l'Asie de l'Est. Les prochains numéros de la revue nous en diront sans doute davantage sur sa diversité.

Soulisack Luanglad CEDICLEC/CELEC, doctorant à l'Université Jean Monnet-Saint-Étienne 